\title{
La evaluación formativa: una herramienta para el desarrollo del pensamiento variacional
}

Andrea Liliana Alarcón Bayona * Claudia Yanneth García Miranda ** Omaida Sepúlveda Delgado ${ }^{* * * *}$

Artículo de reflexión

Fecha de Recepción: 6 septiembre 2017.

Fecha de Aprobación: 4 febrero 2018.

\section{Resumen}

El artículo se presenta la investigación realizada en dos instituciones educativas rurales (Institución Educativa 1, Institución Educativa 2) de Boyacá, con estudiantes del grado $5^{\circ}$ de Básica Primaria quienes presentaron dificultades en pruebas SABER, en el pensamiento variacional y procesos de modelación, comunicación y resolución de problemas, originando la pregunta ¿Las estrategias de evaluación formativa, potencian el desarrollo del pensamiento variacional?; planteándose como objetivo: diseñar e incorporar estrategias de evaluación formativa que desarrollen este pensamiento y los procesos, mediante el diseño, implementación y evaluación de la unidad didáctica "Razones y proporciones".

La metodología de Investigación Acción (Elliott, 1993); determinó las fases de planificación, acción, observación y reflexión. La muestra seleccionada por conveniencia, correspondió a 26 estudiantes de las dos instituciones educativas (IE). El enfoque mixto combinó el método cuantitativo para
*Institución Educativa Jorge Eliécer Gaitán., Boyacá (Colombia)

andreaalarcon1024@gmail. com

** Institución Educativa Suse, Boyacá (Colombia) cayis2123@hotmail.com *** Universidad Pedagógica y Tecnológica de Colombia, Boyacá (Colombia) omaida.sepulveda@uptc. edu.co

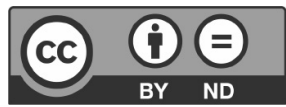


evidenciar el seguimiento al proceso de aprendizaje de los estudiantes y el cualitativo respecto al uso de la Evaluación Formativa, como herramienta para desarrollar el pensamiento variacional. Los resultados establecieron, que la evaluación formativa permitió a los estudiantes reconocer sus aprendizajes y dificultades en: razón, proporcionalidad directa y regla de tres, al vincularlos en actividades generando compromiso $\mathrm{y}$ motivación; y a los docentes, los llevó a plantearse estrategias de enseñanza, atendiendo a las diferencias y necesidades de los estudiantes.

Palabras clave: evaluación, formativa, matemáticas, estrategias, procesos. 


\section{Introducción}

El presente estudio surgió del análisis de las pruebas SABER (2016) e Informes por Colegios de las Instituciones Educativas Jorge Eliécer Gaitán (IE1) y Suse (IE2), en las cuales se evidenciaron debilidades en el componente del pensamiento variacional $y$ en los procesos matemáticos decomunicación, modelación y resolución de problemas (ICFES, 2015). De igual forma, de la observación realizada a las prácticas docentes, donde se constató la ausencia de estrategias de evaluación formativa, para el seguimiento de aprendizajes de los estudiantes; esto se reflejó en los instrumentos de acompañamiento en el aula que se encontraban sistematizados por el Programa Todos a Aprender (PTA), para los años 2015 a 2017. En el Informe por Colegio dado a la IE1, el $78 \%$ de los estudiantes no usaban operaciones, ni las propiedades de los números naturales para establecer relaciones entre ellos, en situaciones específicas. En la IE2, el $83 \%$ de los estudiantes no resolvían, ni formulaban problemas sencillos de proporcionalidad directa (ICFES, 2016).

En la misma dirección, en la IE1 e IE2, el proceso evaluativo del área de matemáticas, ha sido vertical, teniendo en cuenta que el profesor es quien organiza las pruebas que aplica a los estudiantes para medir sus conocimientos y generalmente es realizada de forma escrita, con formatos cerrados; los cuales, según su criterio, dan cuenta del aprendizaje logrado o no, por los estudiantes.
Según Moreno y Ortiz (2008):

Las evaluaciones en matemática se hacen a través de prueba, las cuales son elaboradas por los profesores de acuerdo con los contenidos planificados. Se refleja la connotación cuantitativa que le dan los profesores a la evaluación en Matemática, por cuanto consideran que se realiza para colocar una nota. La evaluación por objetivos se usa para verificar el logro de estos objetivos planeados (p. 150).

Del análisis realizado, emergió la pregunta de investigación: ¿Las estrategias de Evaluación Formativa, potencian el desarrollo del pensamiento variacional? Desde esta perspectiva, se propuso como objetivo general para el estudio: Diseñar e incorporar estrategias de evaluación formativa para el desarrollo del pensamiento variacional y los procesos de comunicación, modelación y resolución de problemas, de los estudiantes de grado quinto; mediante el diseño e implementación de una unidad de matemáticas basada en la proporcionalidad, atendiendo a los objetivos específicos planteados en este estudio, diagnosticando las estrategias de evaluación que los docentes aplican en el aula de clase, realizando un análisis didáctico a priori para el desarrollo del pensamiento variacional, implementando estrategias de evaluación formativa en la unidad didáctica de Matemáticas y reflexionando sobre el efecto del análisis didáctico en la unidad de proporcionalidad; así como de los instrumentos de evaluación, procesos y facetas incorporadas.

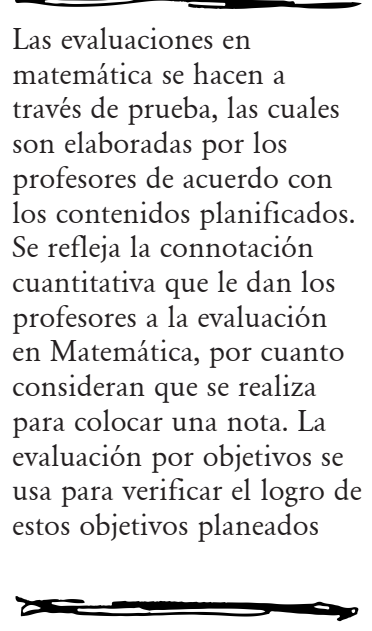

Las evaluaciones en matemática se hacen a través de prueba, las cuales son elaboradas por los profesores de acuerdo con los contenidos planificados. Se refleja la connotación cuantitativa que le dan los profesores a la evaluación en Matemática, por cuanto consideran que se realiza para colocar una nota. La evaluación por objetivos se usa para verificar el logro de estos objetivos planeados 
Para el presente estudio, se empleó la metodología de Investigación Acción, propuesta por Elliott(1993), atendiendo al desarrollo de las fases de planificación, acción, observación y reflexión. Se propuso como estrategia didáctica, la incorporación de la evaluación formativa como una herramienta que permite el fortalecimiento del pensamiento variacional y el desarrollo de los procesos de comunicación, modelación y resolución de problemas, bajo el diseño de una unidad didáctica de matemáticas desarrollada con los estudiantes del grado $5^{\circ}$ de Educación Básica Primaria (EBP).

\section{La Investigación Acción permitió:}

En primer lugar, para el desarrollo de la fase de planificación, se elaboró y validó un cuestionario piloto, con el fin de indagar sobre las estrategias de evaluación formativa implementadas por los docentes de las instituciones educativas. El cuestionario fue validado por profesores disciplinares en el campo de la Didáctica de la Matemática. En segundo lugar, en la fase de acción, se diseñó y ejecutó el análisis didáctico a priori de una unidad de matemáticas en proporcionalidad, siguiendo los lineamientos de los referentes curriculares y la inclusión de las facetas del Enfoque Ontosemiótico (EOS) propuesto por Godino (2009); así como el planteamiento de actividades para el fortalecimiento de los procesos de modelación, comunicación y resolución de problemas, que dieron origen a la elaboración e incorporación de instrumentos de evaluación formativa.
En tercer lugar, la fase de la observación fue de tipo participante y los resultados evidenciados en los instrumentos evaluativos, permitieron consolidar y analizar datos para la realimentación de procesos reflejados en los estudiantes y el planeamiento de la siguiente sesión de clase. En cuarto y último lugar, en la fase de la reflexión se efectuó un análisis a posteriori del desarrollo de las sesiones de clase, acorde con la estrategia de evaluación formativa implementada, facetas ejecutadas y procesos matemáticos de los estudiantes.

Respecto al enfoque del estudio, según Hernández, Fernández y Baptista (2010), es mixto, ya que se analizaron datos cuantitativos y cualitativos, que fueron recolectados en cada una de las fases de la Investigación Acción. En relación con los referentes teóricos del estudio, se articularon los ejes temáticos de Evaluación Formativa (técnicas, instrumentos y modalidades), los referentes curriculares del pensamiento matemático respecto al pensamiento variacional y los procesos matemáticos, siguiendo los Lineamientos Curriculares (LC), los Estándares Básicos de Competencias (EBC), las Matrices de Referencia (MR), Derechos Básicos de Aprendizaje (DBA) y el estudio del Conocimiento Didáctico del Profesor desde el Enfoque Ontosemiótico del conocimiento y la instrucción matemática (EOS), según el modelo propuesto por Godino (2009).

Según los resultados del estudio realizado, se evidenció que la evaluación formativa permitió fortalecer el 
proceso de comunicación, mediante la discusión y la experimentación por parte de los estudiantes, lo cual facilitó el intercambio de saberes. La representación de datos en tablas, permitió la comunicación de los conocimientos adquiridos en otro lenguaje. En cuanto al proceso de modelación, se verificó que el incluir material concreto, representaciones pictóricas y simbólicas y la elaboración de los esquemas conceptuales, posibilitó en los estudiantes la contextualización entre lo real y lo simbólico. Finalmente, respecto al proceso de resolución de problemas, se estableció que el planteamiento de situaciones contextualizadas despertó el interés de los estudiantes. Con relación al estudio del Conocimiento del Profesor (Godino, 2009), se concluyó que el análisis de las facetas del conocimiento didáctico matemático del docente, hace que el profesor promueva en los estudiantes la exploración de saberes, fomente valores, propicie las interacciones en el aula, haga uso de materiales y llegue a la innovación en sus prácticas educativas, desde el desarrollo de un conocimiento disciplinar más profundo.

\section{Referentes teóricos}

\section{Evaluación formativa.}

La práctica de la evaluación ocupa un lugar privilegiado en las Instituciones Educativas, ya que permite indagar en el proceso de aprendizaje de los estudiantes $y$, por tanto, constituye una herramienta fundamental para la gestión educativa. De acuerdo con Condemarín y Medina (2000, p. 15): "la evaluación formativa es un proceso que realimenta el aprendizaje, posibilitando su regulación por parte del estudiante. De esta manera, él junto al educador, puede ajustar la progresión de los aprendizajes y adaptar las actividades de aprendizaje de acuerdo con sus necesidades y posibilidades".

Este tipo de evaluación, direcciona el proceso de enseñanza aprendizaje en la búsqueda del cumplimiento de objetivos y del desarrollo de las competencias en cada sesión de clase, ya que permite la recolección de información, determina el estado de avance y/o dificultad de los estudiantes. Según Castillo \& Cabrerizo (2010), la evaluación formativa:

Sirve como estrategia de mejora para ajustar y regular sobre la marcha los procesos educativos, de cara a conseguir los objetivos previstos y las competencias básicas establecidas. Es la más adecuada para la evaluación de los procesos y suele relacionarse con la evaluación continua. La evaluación formativa permite obtener información de todos los elementos que configuran el desarrollo del proceso educativo de todos y cada uno de los alumnos a lo largo del curso, y permite reorientar, modificar, regular, reforzar, comprobar, etc., los aprendizajes, dependiendo de cada caso particular (p. 38).

La evaluación formativa, conduce al desarrollo de las competencias, para lo cual el docente, selecciona y planea sus prácticas e instrumentos, en forma que le permitan recolectar la mayor información posible durante "la evaluación formativa es un proceso que realimenta el aprendizaje, posibilitando su regulación por parte del estudiante. De esta manera, él junto al educador, puede ajustar la progresión de los aprendizajes y adaptar las actividades de aprendizaje de acuerdo con sus necesidades y posibilidades" 
el proceso de enseñanza, haciendo uso de técnicas e instrumentos. De acuerdo con Castillo \& Cabrerizo (2010):

La técnica se relaciona con el método operativo de carácter general que permite poner en juego distintos procesos o estrategias para obtener la información que se desea; suele utilizar varios instrumentos. En este contexto, el instrumento corresponde a una herramienta específica, un recurso concreto, o un material estructurado que se aplica para recoger la información que se desea y suele estar vinculado a una técnica (p. 328).

En el estudio, se dio relevancia a las listas de control, que, según Castillo \& Cabrerizo (2010):

Son técnicas de observación: se centran en registrar la aparición o no de una conducta durante el periodo observado, pero sin delimitar su frecuencia o duración. El instrumento que utilizan es muy simple: una tabla donde se recogen las conductas a observar y dos posibles opciones (síno). Previo a la observación, el profesor ha debido especificar aquellas conductas a evaluar (p.362).

Tabla 1.

Técnicas e instrumentos de evaluación.

\begin{tabular}{ll}
\hline Técnicas & Instrumentos \\
\hline De observación & -Sistema de categorías \\
& -Listas de control \\
& -Registro anecdótico \\
& -Diario de aula \\
De & -Cuestionarios \\
interrogación & $\begin{array}{l}\text {-Exámenes } \\
\text {-Pruebas objetivas }\end{array}$ \\
Otras técnicas & -Portfolio \\
& -Rúbricas \\
\hline
\end{tabular}

Fuente: Castillo \& Cabrerizo, (2010, p.216).
Entre los instrumentos se encuentra el Diario, donde se transcriben los registros retrospectivos, de los eventos y sujetos: contienen repetidas observaciones sobre un individuo o grupo. En la misma línea, las Pruebas objetivas: "Son instrumentos de evaluación que permiten medir el grado de consecución alcanzado por cada alumno en relación con los criterios establecidos previamente y desarrollados en la programación general” (p.387).

Entre las modalidades de evaluación utilizadas, se encuentran: la autoevaluación, como el proceso por el cual se participa en la propia valoración de la actividad escolar: fue de gran utilidad ya que permitió que el estudiante identificara sus avances, logros y dificultades: le posibilitó reflexionar sobre su quehacer, tanto individual como cooperativo, dentro del proceso educativo, de igual forma el llegar a asumir una postura críticoreflexivo y a autorregular su proceso de aprendizaje. Respecto al docente, estas modalidades de evaluación, lo llevaron al reconocimiento de factores objetivos para enriquecer y reorientar a sus estudiantes, valorar su actuación y, de igual forma, lo direccionaron a un conocimiento más cercano de su grupo, y le permitieron ajustar y realimentar los ejes temáticos, las estrategias de enseñanza y los programas en general. Bajo esta mirada, se utilizó la coevaluación, entendida como la evaluación entre pares, la cual permitió a los estudiantes la reflexión sobre los avances y dificultades individuales $\mathrm{y}$ grupales. 


\section{Referentes curriculares}

Para el estudio del pensamiento variacional en Matemáticas, se tomó como referencia los Lineamientos Curriculares del área de matemáticas (LCM, 1998), los Estándares Básicos de Competencias (EBC, 2006), las Matrices de Referencia (DBA, 2016) y los Derechos Básicos de Aprendizaje (DBA, 2016). Los LCM (1998), plantean aspectos fundamentales para organizar el currículo, desde los conocimientos básicos a partir de los cinco pensamientos matemáticos: el numérico, que se relaciona con los sistemas numéricos; el variacional, con los sistemas algebraicos; el aleatorio, con los sistemas de datos; el espacial, con los sistemas geométricos; y el pensamiento métrico, con los sistemas de medidas.

Para el desarrollo del estudio, se abordó el pensamiento variacional en situaciones de variación y cambio. Este pensamiento, tiene que ver con el reconocimiento, la percepción, la identificación y la caracterización de la variación y el cambio en diferentes contextos, así como su descripción, modelación y representación en distintos sistemas o registros simbólicos, ya sean verbales, icónicos, gráficos y algebraicos (EBC, 2006). En este sentido, los Lineamientos curriculares (1998) proponen que:

El estudio de la variación puede ser iniciado pronto en el currículo de matemáticas. El significado y sentido acerca de la variación puede establecerse a partir de las situaciones problemáticas cuyos escenarios sean los referidos a fenómenos de cambio y variación de la vida práctica. (LCM, 1998, p. 73).

De acuerdo con los EBC (2006), el desarrollo del pensamiento variacional:

Se inicia con el estudio de las regularidades y la detección de criterios que rigen esas regularidades o las reglas de formación para identificar el patrón que se repite periódicamente. Las regularidades (entendidas como unidades de repetición) se encuentran en sucesiones o secuencias que presentan objetos, sucesos, formas o sonidos, en un orden fijado o de acuerdo a un patrón. De esta manera, la unidad que se repite con regularidad da lugar a un patrón. Al identificar en qué se parecen y en qué se diferencian los términos de estas sucesiones o secuencias: se desarrolla la capacidad para identificar en qué consiste la repetición del mismo patrón y la capacidad para reproducirlo por medio de un cierto procedimiento, algoritmo o fórmula. (p.66).

Los LCM (1998) y EBC (2006) enuncian los procesos generales, que trazan el recorrido de los aprendizajes de los estudiantes; dentro de estos, se encuentran: la elaboración, comparación y ejercitación de procedimientos, la comunicación, modelación, razonamiento y resolución de problemas. Para el estudio, se abordaron los procesos de comunicación, modelación y resolución de problemas. El proceso de comunicación, según los EBC (2006), 
puede ocurrir cuando los estudiantes trabajan en grupos cooperativos, cuando un estudiante explica un algoritmo para resolver ecuaciones, cuando un estudiante presenta un método único para resolver un problema, cuando el estudiante construye y explica una representación gráfica de un fenómeno del mundo real, o cuando el estudiante propone una conjetura sobre una figura geométrica (EBC, 2006).

Frente al proceso de la modelación, se establece que el estudiante aprende matemáticas haciendo matemáticas (EBC, 2006, p. 97), para lo cual debe partir de situaciones problema de la vida diaria y relacionarlas con su contexto para su comprensión y formulación, de manera que puedan transferir dicha situación por medio de actividades como: Identificar las matemáticas en un contexto general, esquematizar, formular y visualizar un problema de diferentes formas, descubrir relaciones y regularidades, transferir un problema de la vida real a un problema matemático o viceversa (EBC, 2006, p. 99).

Según los LCM (1998), "el sentido de la variación puede establecerse a partir de situaciones problemáticas cuyos escenarios son referidos a fenómenos de variación y de la vida práctica" (p.73). En esta dirección, se aborda la resolución de problemas, la cual:

requiere el desarrollo de una actividad mental, para que a partir de una serie de estrategias, los estudiantes puedan resolver, encontrar resultados, verificar, interpretar, modificar y plantear ejercicios abiertos, que permitan al estudiante la elección de múltiples respuestas (EBC, 2006, p. 52).

Respecto al contexto, se relaciona con el ambiente que rodea al estudiante y que le da sentido a las matemáticas que aprende. Es allí donde entra en juego el rol del docente para generar situaciones problemáticas que involucran la afectividad del estudiante. En este sentido, la situación problemática se convierte en un microambiente de aprendizaje que puede provenir de la vida cotidiana, de las matemáticas y de las otras ciencias (EBC, 2006, p.52).

En la misma dirección, se abordaron las matrices de referencia, las cuales representan los aprendizajes que evalúa el ICFES (2016), en las pruebas Saber respecto a cada competencia, relacionándolos con las evidencias que debería hacer y manifestar el estudiante que haya logrado dichos aprendizajes en la componente (pensamiento) y la competencia específica (proceso) (EBC, 2006, p.2).

Finalmente, se enunciaron los Derechos Básicos de Aprendizaje (DBA, 2016), los cuales corresponden al conjunto de saberes que debe apropiar el estudiante al finalizar cada grado y en un área en particular.

\section{El Conocimiento Didáctico- Matemático del profesor}

El profesor articula diversos componentes para la ejecución de la enseñanza y para el logro del aprendizaje; esto lleva a constituir un 
conocimiento didáctico del contenido del profesor. Desde este punto de vista, el enfoque teórico en matemáticas EOS, sirve de herramienta para el análisis y la reflexión de los procesos de enseñanza y aprendizaje de las matemáticas. Bajo esta perspectiva, el Conocimiento Didáctico Matemático del profesor, se encuentra constituido por facetas o dimensiones, que sirven para planificar e implementar procesos de instrucción en matemática. De acuerdo con Godino (2009):

La faceta epistémica, se relaciona con el contexto institucional y la distribución del contenido; la faceta cognitiva, se enfoca al conocimiento personal de los estudiantes y al desarrollo de los significados personales en el aprendizaje; en la dimensión afectiva, intervienen las actitudes, creencias y valores de los estudiantes; la faceta mediacional, se relaciona con los recursos tecnológicos y con el tiempo para la instrucción; la faceta interaccional, permite determinar patrones de interacción y la secuenciación que se da en la construcción del conocimiento. Finalmente, se tiene la faceta ecológica, la cual se relaciona con el entorno socio político, económico y cultural del estudiante (p.21).

\section{Metodología}

La metodología utilizada en el estudio, correspondió a la Investigación Acción sustentada en Elliott (1993), la cual integró las fases de planificación, acción, observación y reflexión. La planificación, para identificar la dinámica evaluativa efectuada por los docentes y en la realización del análisis del cuestionario piloto, aplicado a los estudiantes del grado $5^{\circ}$ de las instituciones educativas. La acción, para diseñar y ejecutar el análisis didáctico a priori de la unidad de proporcionalidad: este análisis didáctico se realizó desde el Enfoque Ontosemiótico del conocimiento y la instrucción matemática (EOS) con el planteamiento de una situación matemática denominada "Fiesta matemática", que surgió de un ambiente contextualizado como es la celebración del día del estudiante, en donde los participantes desarrollaron actividades relacionadas con la razón, proporcionalidad directa y regla de tres, con uso de material concreto, pictórico y simbólico para determinar el número de masmelos y gomitas para la elaboración de pasabocas (razones); la cantidad de ingredientes para preparar las tortas requeridas para 100 personas (proporción); la cantidad de buses para el desplazamiento de los estudiantes con destino a Playa Blanca (proporcionalidad directa); el costo de los pasajes y sus entradas (regla de tres).

Cada sesión de clase partió de la exploración de saberes previos conducentes a superar dificultades y constituyó el punto de partida de la nueva temática a abordar, con ejemplificaciones concretas para llegar a la adquisición del conocimiento.

Finalmente, en cada sesión se implementaron estrategias de evaluación formativa que, en su orden, fueron: lista de control, diario de clase, autoevaluación y rúbrica;
La faceta epistémica, se relaciona con el contexto institucional y la distribución del contenido; la faceta cognitiva, se enfoca al conocimiento personal de los estudiantes y al desarrollo de los significados personales en el aprendizaje 
así como de una prueba objetiva que permitió determinar dificultades, realizar un seguimiento y realimentar los procesos de enseñanza aprendizaje. La observación, mediante la técnica de observación participante y la ayuda de instrumentos evaluativos para recoger y analizar datos. La reflexión, se realizó a través del análisis a posteriori, según el desarrollo de las sesiones de clase y las estrategias de evaluación formativa empleadas, facetas ejecutadas y en el análisis del desarrollo de los procesos matemáticos.

\section{Resultados y discusiones}

\section{Fase de planificación.}

\section{Categoría de Evaluación Formativa.}

En un primer momento, se evidenció en los cuestionarios aplicados a los estudiantes del grado $5^{\circ}$ de las dos IE, un desconocimiento de los elementos relevantes de la evaluación formativa para el proceso de aprendizaje. Al preguntar a los estudiantes, sobre las sugerencias de los docentes para mejorar sus aprendizajes, se vislumbró que faltaban acciones para promover el descubrimiento y la corrección de los errores. Igualmente, se estableció que no se daban a conocer los criterios de evaluación, para identificar el desempeño del estudiante. En la IE1, el $36 \%$ de los estudiantes no sabían lo que el docente les evaluaba en las sesiones de clase: y, de igual forma, en la IE2, donde el porcentaje corresponde al $55 \%$. Al indagar sobre la frecuencia con la que se implementaban las estrategias de evaluación formativa en el aula, en la IE1 y en la IE2, el 71\% y 55\% de los estudiantes respectivamente, manifestaron que nunca y muy pocas veces se incorporaban estos instrumentos al aula. Frente a las modalidades de evaluación, se resalta el hecho que en la IE1 el 93\% y en la IE2 el 80\% de los estudiantes muy pocas veces o nunca han realizado una autoevaluación. Respecto de la coevaluación, en la IE1 el 57\% de los estudiantes nunca o muy pocas veces la habían realizado; y en la IE2, este porcentaje corresponde al 91\%.

\section{Categoría de procesos} matemáticos.

En el proceso de comunicación, se evidenció que, en las sesiones de clase de matemáticas, se ignoraba en algunos casos este proceso fundamental, donde los estudiantes leen e interpretan conceptos a través de gráficos, palabras, tablas y representaciones; lo cual permitió desarrollar las habilidades necesarias para contextualizar los conocimientos y expresarlos de diversas formas. Según el cuestionario, en la IE1 el 71\% de los estudiantes no hacen uso de dibujos, tablas, esquemas para desarrollar un ejercicio matemático; y en la IE2, este porcentaje corresponde al $36 \%$. En el proceso de modelación, en cuanto a la inclusión de material real y manipulativo dentro de las sesiones de clase donde el estudiante puede esquematizar, visualizar y dar solución a problemas de diferentes formas, se estableció que esta situación no se estaba vivenciando en el aula; dado que en la IE1 el 93\% de los estudiantes enunció que nunca y muy pocas veces, se emplearon este tipo de materiales en 
el aula para la solución a una situación problema: en la misma dirección en la IE2, con un porcentaje del 73\%.

\section{Fase de Acción}

Atendiendo a la fase de planificación y al análisis de los resultados de las Pruebas SABER e Informes por Colegio 2016 de las IE objeto de la investigación, se estableció la necesidad de elaborar una unidad con el planteamiento de una situación problema contextualizadora, titulada "Fiesta Matemática", para abordarla en cuatro sesiones de clase desde el EOS, buscando la potenciación y el desarrollo del pensamiento variacional y centrada en el eje temático de la proporcionalidad. Las sesiones se establecieron de la siguiente manera: Sesión 1. Razón; Sesión 2. Proporcionalidad; Sesión 3. Proporcionalidad directa; y Sesión 4. Realimentación proporcionalidad directa. En cada sesión, la evaluación formativa cobró sentido al ser una herramienta fundamental soportada en los instrumentos de evaluación formativa, los cuales se constituyen en medios facilitadores para optimizar los aprendizajes de los estudiantes.

\section{Fase de observación}

Categoría de Evaluación Formativa.

Durante las cuatro sesiones de clase, se emplearon instrumentos de evaluación, como: Sesión 1: Lista de control para las dos IE, que permitió evidenciar las representaciones en lenguaje gráfico y simbólico para adquirir el concepto de razón y la solución a una situación problema relacionada con el concepto. Tanto en la IE1 como en la IE2, los estudiantes en su mayoría, presentaron falencia para establecer patrones de secuencia adecuados y representarlos mediante tablas. En la sesión 2, se utilizó el diario de clases donde se constató que los estudiantes de las IE1 e IE2, hacen uso adecuado de material concreto para resolver una situación descrita, el planteamiento de proporciones de manera apropiada por parte de la mayoría de estudiantes, definición y resolución de la tarea de la semana 2 y desarrollo de la prueba individual con eficacia por la mayoría de estudiantes. En las instituciones objeto de estudio, se estableció que los estudiantes, tanto en la IE1 como en la IE2, presentaron conflictos semióticos o de significado en los procesos de operaciones básicas de adición, sustracción, multiplicación y división.

En la sesión 3, los estudiantes de la IE1 y de la IE2 expresaron en su autoevaluación, el empleo adecuado de siluetas para representar situaciones, participación dinámica en las actividades y realización de aportes para solución de situaciones. En las Instituciones objeto de estudio, se estableció que los estudiantes presentaron dificultad para adquirir el concepto de proporcionalidad directa evidenciado al no encontrar el término desconocido de la proporción o "cuarto término" y respecto a la regla de tres, se reflejó falencia en el despeje de términos y seguimiento correcto de los pasos para dar solución a las preguntas formuladas.

En la sesión 4, en las IE objeto de estudio, se vislumbró mediante las 
rúbricas que la mayoría de estudiantes realizaron aportes para dar respuestas a preguntas relacionadas con el video sobre proporcionalidad directa. En la misma dirección, se percibe excelente participación de los estudiantes en la formación de conjuntos y atendiendo a un patrón de secuencia que se evidenció en la repetición de una figura o número, en un orden específico construido mediante actividad lúdica. De igual forma, se analizó la dificultad para reconocer el concepto de proporcionalidad directa y para emplear la regla de tres.

\section{Categoría procesos de pensamiento.}

\section{Modelación.}

Los estudiantes de las dos instituciones modelaron adecuadamente pinchos con material real, crearon cuadrados con palillos, atendiendo a un patrón establecido (ser cuadrado de). Los estudiantes hicieron uso de siluetas de buses y personas de manera adecuada, con el fin de dar respuesta a la primera tarea de la situación problema correspondiente a la tercera semana. Los estudiantes emplearon material concreto para formar conjuntos, estableciendo relaciones de proporcionalidad directa. En la IE1 y en la IE2, se observó que los estudiantes tienen debilidad para definir la tarea a resolver en una situación Matemática descrita anteriormente.

\section{Comunicación.}

En la IE1 y en la IE2, los estudiantes evidenciaron participación en la solución de interrogantes planteados y aportes a nivel grupal, traducción de situaciones concretas de forma pictórica, intercambio de ideas y conocimientos, y representación de información en tablas desde la sesión 2. Así mismo, en las instituciones objeto de estudio se notó en los estudiantes debilidad para determinar un patrón de secuencia y expresarlo en tablas durante la sesión 1 .

\section{Resolución de problemas.}

La mayoría de estudiantes (19 de 25) lograron establecer razones a partir de una receta, 16 de los 25 estudiantes lograron establecer la cantidad de ingredientes para elaborar la torta mediante proporcionalidad directa por secuenciación y algunos mediante regla de tres. Los estudiantes siguieron ejemplos para afianzar el concepto de proporcionalidad directa y regla de tres. Se estableció que 15 de los 25 estudiantes, resuelven situaciones aplicando pasos de la regla de tres. Algunos estudiantes de las dos Instituciones Educativas presentaron dificultades para establecer patrones de cantidades de dos o más dígitos, 9 de los 25 estudiantes demostraron conflictos semióticos para seguir los pasos de la regla de tres, al resolver una situación problema.

\section{Categoría facetas del EOS.}

\section{Faceta Cognitiva.}

Se verificó la conceptualización en los estudiantes de las 2IE acerca de los temas de razón, proporciones y proporcionalidad directa mediante 
lectura e interpretación de mapas conceptuales y la dificultad en el planteamiento y seguimiento de los pasos de la regla de tres.

\section{Faceta mediacional.}

Se constató que el uso de material concreto (billetes, siluetas, recursos tecnológicos y elementos del entorno) permitió la modelación de situaciones para adquirir aprendizajes desde lo concreto-pictórico-abstracto.

\section{Faceta interaccional.}

Se observó el diálogo entre los estudiantes e interacción con el docente; situación que permitió que se identificaran conflictos semióticos por parte de los estudiantes en procesos algorítmicos o simbólicos; así como constatar la poca participación de algunos estudiantes en el trabajo cooperativo. No obstante, se verificó el desarrollo efectivo de tareas a nivel grupal.

\section{Faceta ecológica.}

En la IE1 e IE2, la innovación con propuestas de situaciones matemáticas contextualizadas y acordes a los intereses de los estudiantes y al entorno escolar, fue motivante y enriquecedora en el desarrollo de los objetivos propuestos.

\section{Faceta afectiva.}

Se reconoció la interacción entre los estudiantes: respeto de sus puntos de vista, responsabilidad de cada estudiante en el rol asignado para el desarrollo de aprendizajes cooperativos; además, cumplimiento de las actividades propuestas en la guía y las actitudes de empatía entre ellos, dio cuenta de ambientes efectivos de aprendizaje en el grupo objeto de este estudio.

\section{Faceta epistémica.}

El lenguaje matemático relacionado con esta faceta, fue abordado en las sesiones implementadas a través de situaciones problemas, lenguajes (términos, expresiones, notaciones, gráficos); acciones, conceptos y argumentaciones; estableciéndose las siguientes conclusiones en cada una de ellas:

Para las situaciones problema, en el análisis a priori se diseñó una situación generadora y motivacional referida con el entorno escolar, que fomentó la participación y los conocimientos de los estudiantes hacia el eje temático de la proporcionalidad y conducente al logro de los objetivos propuestos. De igual forma, los estudiantes pudieron desarrollar y potenciar los conocimientos en pensamiento variacional y procesos matemáticos.

En relación con el lenguaje y la función que cumplen los términos de las razones y las proporciones, fueron identificados por los estudiantes en las diferentes sesiones de clase. En cuanto a expresiones y notaciones, los estudiantes incorporaron expresiones referentes a razones y proporciones dadas en las sesiones y de tipo a: b (a es a b) y a/b para la aplicación en situaciones matemáticas.

En lo referente a gráficos, los estudiantes elaboraron esquemas 
Finalmente, los conceptos referidos a razones, proporciones, proporcionalidad directa y regla de tres, fueron adquiridos por los estudiantes de comprensión para identificar la información y la tarea a desarrollar. En el mismo sentido, leyeron, analizaron e interpretaron esquemas para definir las tareas de cada una de las semanas.

Otro elemento de esta faceta son las acciones, donde los estudiantes respondieron preguntas de forma oral y escrita, participaron en actividades de trabajo cooperativo en cada una de las sesiones y realizaron las actividades planteadas siguiendo las indicaciones descritas en la guía propuesta.

Finalmente, los conceptos referidos a razones, proporciones, proporcionalidad directa y regla de tres, fueron adquiridos por los estudiantes, mediante las actividades presentadas en las guías y con refuerzo por medio de mapas conceptuales y ejemplos guías; además, de la explicación proporcionada por las docentes.

\section{Fase de reflexión.}

La ejecución del proyecto llevó a los estudiantes a:

- La contextualización de la situación problema, lo cual cambió la percepción de los estudiantes sobre la Matemática, descubriendo su aplicabilidad en situaciones contextualizadas (fiesta matemática) mediante la acción planificada de un evento a desarrollar (celebración día del estudiante), la exploración de material concreto, pictórico y simbólico para dar respuesta a interrogantes planteados; la sistematización con la construcción de los conceptos tratados, la confrontación mediante la verificación de conocimientos adquiridos en grupoy, posteriormente, las orientaciones dadas por el docente, el debate en la discusión generada por los estudiantes en el trabajo grupal, la evaluación mediante pruebas objetivas permitiendo reconocer sus aprendizajes y la autoevaluación y heteroevaluación mediante los instrumentos utilizados para tal fin; como lo enuncian Obando \& Muñera (2003):

Una situación problema la podemos interpretar como un contexto de participación colectiva para el aprendizaje, en el que los estudiantes, al interactuar entre ellos mismos, y con el profesor, a través del objeto de conocimiento, dinamizan su actividad matemática, generando procesos conducentes a la construcción de nuevos conocimientos. Así, ella debe permitir la acción, la exploración, la sistematización, la confrontación, el debate, la evaluación, la autoevaluación, la heteroevaluación (p. 185).

- El seguimiento continuo del desarrollo de los procesos de los estudiantes y de las facetas abordadas, donde la evaluación tiene un carácter eminentemente procesal, tal modalidad es orientadora y no prescriptiva, dinámica y marcha paralelamente con los objetivos o propósitos que pautan la instrucción (Rotger, 1990, p.391).

- La modelación de situaciones por parte de los estudiantes para su abstracción como lo fundamenta Guzmán (1993): 
La manipulación de objetos, desarrollo de la capacidad mental, planteamiento de reflexiones, realización de transferencias, fortalecimiento de la confianza, actividades lúdicas que promuevan el interés de los niños y la preparación de los estudiantes para enfrentarse a problemas de la ciencia, de la vida y de los nuevos retos de la tecnología (p.111).

- El diseño de guías para el estudiante, permitió la construcción del conocimiento inductivo con la orientación del docente en el tema de proporcionalidad. Según; Godino (2002), se debe proporcionar una amplia variedad de tareas sobre razones y proporciones en diversos contextos que pongan en juego relaciones multiplicativas entre distintas magnitudes (p.434).

- Se logró, la argumentación, las contraposiciones, el intercambio de ideas, las ejemplificaciones, como mecanismos para fortalecer los aprendizajes de los estudiantes. De acuerdo con Godino (2002):

Se debe estimular la discusión y la experimentación en la comparación y predicción de razones; procurar que los niños distingan las situaciones de comparación multiplicativa (proporcionalidad) de las no multiplicativas, proporcionando ejemplos y discutiendo las diferencias entre ellas (p. 432).

Con el desarrollo del proyecto, los docentes reflexionaron sobre:

- El fortalecimiento disciplinar y didáctico a través de los análisis a priori y a posteriori y el diseño de las clases a partir de las dificultades evidenciadas con base en los instrumentos de evaluación formativa; ya que es primordial en el proceso, plantear objetivos específicos y adecuar el ambiente de la evaluación con las mejores condiciones, a partir de las habilidades del estudiante y con los tiempos requeridos para el cumplimiento de la tarea en clase, como lo expresan Chadwick \& Rivera (1991).

- El reconocimiento de la proporcionalidad como un objeto complejo que requiere de actividades de manipulación y representación, antes de llegar al lenguaje simbólico y de acuerdo con Godino (2002):

Se debe reconocer que los métodos mecánicos de manipulación de símbolos, como los esquemas del tipo de regla de tres, para resolver problemas de proporcionalidad no son apropiados para desarrollar el razonamiento proporcional y no se deberían introducir hasta que los alumnos tengan un cierto dominio de otros métodos intuitivos y con un fundamento matemático consistente (p. 434).

\section{Conclusiones}

Atendiendo a los objetivos definidos para este estudio, se concluye que:

Con el diagnóstico dentro de la fase de planificación y a partir de los resultados del cuestionario; se percibió que los docentes priorizaban las sugerencias comportamentales sobre las 
pedagógicas; los ejercicios matemáticos carecían de material manipulativo, se desconocía la funcionalidad de dibujos, tablas y esquemas para el desarrollo de los procesos matemáticos; los estudiantes desconocían los objetivos de la evaluación, hacía falta incorporar estos instrumentos de Evaluación Formativa y el uso de otras modalidades de evaluación como autoevaluación y coevaluación.

El análisis didáctico a priori de la unidad de proporcionalidad, según el EOS, facilitó el dominio del conocimiento disciplinar y didáctico, el diseño de acciones concretas en el análisis de las facetas y procesos, permitiendo la construcción de instrumentos de evaluación y guías del estudiante por parte de los docentes; siguiendo los Lineamientos propuestos por el MEN para el diseño de clases innovadoras y contextualizadas que condujeron al desarrollo del pensamiento variacional y la potenciación de procesos de comunicación, modelación y resolución de problemas.
La implementación de estrategias de Evaluación Formativa, fue relevante para identificar las fortalezas $y$ dificultades de los estudiantes, definir acciones de mejora, promover realimentación a los estudiantes y comprometer al docente frente al seguimiento de procesos de aprendizaje en pensamiento variacional, así como la participación de los estudiantes en su proceso valorativo.

Finalmente, el análisis a la implementación de la unidad didáctica de proporcionalidad con la inclusión de instrumentos de Evaluación Formativa, definición de procesos y facetas incorporadas en cada sesión de clase; permitieron establecer que las estrategias de evaluación formativa influyen en el desarrollo y la potenciación del Pensamiento Variacional; ya que son relevantes para establecer las fortalezas y las dificultades de los estudiantes con miras a proponer acciones de mejora oportunas.

\section{Referencias}

Castillo, S., \& Cabrerizo, J. (2010). Evaluación educativa de aprendizajesy competencias. Madrid: Pearson educación, S.A.

Chadwick, C., \& Rivera, N. (1991). Evaluación formativa para el docente. España: Paidós.

Condemarín, M., \& Medina, A. (2000). Evaluación de los Aprendizajes: un medio para mejorar las competencias lingüísticas y comunicativas. (1a.ed.).Chile: Ministerio de Educación.

DBA. (2016). Derechos Básicos de Aprendizaje y Matrices de Referencia. Bogotá: Editorial MEN. 
Elliott, J. (1993). El cambio educativo desde la Investigación Acción. Madrid: Ediciones Morata.

EBC. (2006). Documento No 3: Estándares Básicos de Competencias en Lenguaje, Matemáticas, Ciencias y Ciudadanas. Lo que los estudiantes deben saber y saber hacer con lo que aprenden. Bogotá: Ministerio de Educación Nacional.

Godino, J. (2009). Categorías de Análisis de los conocimientos del Profesor de Matemáticas. Revista Iberoamericana de Educación Matemática. UNIÓN. 13-31.

Godino, J., \& Batanero, C. (2002). Matemáticas y su didáctica para maestros. Manual para el estudiante. Proyecto. Edumat-Maestros. Recuperado en febrero de 2002 de http://www.ugr.es/local/jgodino/edumat-maestros/

Guzmán, M. (1993). Enseñanza de las ciencias y de las matemáticas. Madrid: Editorial Popular.

Hernández, R., Fernández, C., \& Baptista, P. (2010). Metodología de la investigación. (5a․ Ed.). México: McGrawHill / Interamericana Editores, S.A de C.V.

ICFES. (2015). Informe por colegio Pruebas SABER. (2015). $3^{\circ}, 5^{\circ}$ y $9^{\circ}$. Aterrizando los aprendizajes al aula.

ICFES. (2016). Instituto Colombiano para el Fomento de la Educación Superior. Prueba $S A B E R 3^{\circ}, 5^{\circ}$ y $9^{\circ}$.

LCM. (1998). Lineamientos Curriculares de Matemáticas. Ministerio de Educación Nacional.

Moreno, I., \& Ortiz, J. (2008). Docentes de Educación Básica y sus Concepciones acerca de la Evaluación en Matemática. Revista Iberoamericana de Evaluación Educativa, 1(1), 141-150.

Obando, G., \& Muñera, J. (2003). Las situaciones problema como estrategia para la conceptualización matemática. Revista Educación y Pedagogía, (XV), (35), 185-199. Rotger, B. (1990). Evaluación formativa. Madrid: Editorial Cincel. 